\title{
Synchronization of Coupled Stochastic Systems Driven by $\alpha$-Stable Lévy Noises
}

\author{
Anhui Gu \\ College of Science, Guilin University of Technology, Guilin, Guangxi 541004, China \\ Correspondence should be addressed to Anhui Gu; gahui@glut.edu.cn
}

Received 9 October 2012; Revised 25 December 2012; Accepted 28 December 2012

Academic Editor: Weihai Zhang

Copyright ( 2013 Anhui Gu. This is an open access article distributed under the Creative Commons Attribution License, which permits unrestricted use, distribution, and reproduction in any medium, provided the original work is properly cited.

\begin{abstract}
The synchronization of the solutions to coupled stochastic systems of $N$-Marcus stochastic ordinary differential equations which are driven by $\alpha$-stable Lévy noises is investigated $(N \in \mathbb{N}, 1<\alpha<2)$. We obtain the synchronization between two solutions and among different components of solutions under certain dissipative conditions. The synchronous phenomena persist no matter how large the intensity of the environment noises. These results generalize the work of two Marcus canonical equations in X. M. Liu et al.'s $(2010)$.
\end{abstract}

\section{Introduction}

The synchronization of coupled systems is a ubiquitous phenomenon in the biological and physical science and is also known to occur in abundant of social science contexts; see for example [1-6] and references therein. In the recent book of Strogatz [4], a number of its diversity of occurrence and an extensive list of references can be found. Let $u(t), v(t) \in \mathbb{R}^{d}$ be two functions defined in $\left[t_{0}, \infty\right)\left(t_{0} \in \mathbb{R}\right)$ and $u(t), v(t)$ are said to be synchronized if $\lim _{t \rightarrow \infty}\|u(t)-v(t)\|=0$. Synchronization of deterministic coupled systems has been investigated both for autonomous systems and nonautonomous systems (see, e.g., [7-10]). For coupled systems of Itö stochastic differential equations with various Gaussian noises (in the terms of Brownian motion), the synchronization of solutions has been considered in the papers Caraballo and Kloeden [11], Caraballo et al. [12], Caraballo et al. [13] and Chueshov and Schmalfuß [14]. In [15], Shen et al. showed the synchronization of solutions for more general systems with multiplicative noise. Recently, Liu et al. $[16,17]$ studied the synchronization phenomenon for coupled systems driven by non-Gaussian noises (in terms of Lévy motion) and the analogous results also hold for the general systems with additive Lévy noises [18].

A Lévy motion $L_{t}$ is a non-Gaussian process with independent and stationary increments; that is, increments
$\Delta L_{t}=L_{t+\Delta t}-L_{t}$ are stationary and independent for any non overlapping time lags $\Delta t$. Moreover, its sample paths are only continuous in probability, namely, $\mathbb{P}\left(\left|L_{t}-L_{t_{0}}\right| \geq \epsilon\right) \rightarrow 0$ as $t \rightarrow t_{0}$ for any positive $\epsilon$. With a suitable modification, these paths may be taken as càdlàg; that is, paths are continuous on the right and have limits on the left (see, e.g., $[19,20]$ ). As a special case of Lévy processes, the symmetric $\alpha$-stable Lévy motion plays an important role among stable processes just like Brownian motion among Gaussian processes. A stochastic process $\left\{L_{t}, t \geq 0\right\}$ is called the $\alpha$-stable Lévy motion if (1) $L_{0}=0$ a.e., (2) $L$ has independent increments, and (3) $L_{t}-L_{s} \sim \mathbf{S}_{\alpha}\left((t-s)^{1 / \alpha}, \beta, 0\right)$ for $0 \leq s<t<\infty$ and for some $0<\alpha \leq 2,-1 \leq \beta \leq 1$, where $\mathbf{S}_{\alpha}(\sigma, \beta, v)$ denotes the $\alpha$-stable distribution with index of stability $\alpha$, scale parameter $\sigma$, skewness parameter $\beta$, and shift parameter $\nu$; in particular, $\mathbf{S}_{2}(\sigma, 0, \mu)=N\left(\mu, 2 \sigma^{2}\right)$ denotes the Gaussian distribution. For more details on $\alpha$-stable distributions, we can refer to $[21,22]$.

Let $(\Omega, \mathscr{F}, \mathbb{P})$ be a probability space, where $\Omega=D\left(\mathbb{R}, \mathbb{R}^{d}\right)$ of càdlàg functions with the Skorohod metric (see [23]) as the canonical sample space and denote by $\mathscr{F}:=\mathfrak{B}\left(D\left(\mathbb{R}, \mathbb{R}^{d}\right)\right)$ the Borel $\sigma$-algebra on $\Omega$. Let $\mu_{L}$ be the (Lévy) probability measure on $\mathscr{F}$ which is given by the distribution of a twosided Lévy process with paths in $\Omega$, that is, $\omega(t)=L_{t}(\omega)$. Define $\theta=\left(\theta_{t}, t \in \mathbb{R}\right)$ on $\Omega$ the shift by $\left(\theta_{t} \omega\right)(s):=\omega(t+$ 
$s)-\omega(t)$. Then, the mapping $(t, \omega) \rightarrow \theta_{t} \omega$ is continuous and measurable [24], and the (Lévy) probability measure is $\theta$-invariant, that is, $\mu_{L}\left(\theta_{t}^{-1}(A)\right)=\mu_{L}(A)$, for all $A \in \mathscr{F}$; see [19] for more details.

Consider the following Marcus stochastic ordinary differential equations (MSODEs) system driven by $\alpha$-stable Lévy noises in $\mathbb{R}^{d}$ :

$$
d X_{t}^{(j)}=f^{(j)}\left(X_{t}^{(j)}\right) d t+\sum_{i=1}^{m} c_{i}^{(j)} X_{t}^{(j)} \diamond d L_{t}^{(i)}, \quad j=1, \ldots, N
$$

where $c_{i}^{(j)} \in \mathbb{R}, L_{t}^{(i)}$ are independent $\alpha$-stable Lévy noises on $(\Omega, \mathscr{F}, \mathbb{P}), 1<\alpha<2, \diamond$ denotes the Marcus integral (see, e.g., [25]), and $f^{(j)}, j=1, \ldots, N$ are regular enough to ensure the existence and uniqueness of solutions and satisfy the onesided dissipative Lipschitz condition

$$
\begin{array}{r}
\left\langle x_{1}-x_{2}, f^{(j)}\left(x_{1}\right)-f^{(j)}\left(x_{2}\right)\right\rangle \leq-L\left\|x_{1}-x_{2}\right\|^{2}, \\
j=1, \ldots, N,
\end{array}
$$

on $\mathbb{R}^{d}$ for some $L>0$.

Set

$$
x^{(j)}(t, \omega)=e^{-O_{t}^{(j)}} X_{t}^{(j)}(\omega), \quad t \in \mathbb{R}, \omega \in \Omega, j=1, \ldots, N,
$$

where

$$
O_{t}^{(j)}:=O_{t}^{(j)}(\omega)=\sum_{i=1}^{m} c_{i}^{(j)} e^{-t} \int_{-\infty}^{t} e^{s} d L_{s}^{(i)}, \quad j=1, \ldots, N
$$

are the stationary solutions of the Ornstein-Uhlenbeck stochastic differential equations

$$
d O_{t}^{(j)}=-O_{t}^{(j)} d t+\sum_{i=1}^{m} c_{i}^{(j)} \diamond d L_{t}^{(i)}, \quad j=1, \ldots, N
$$

Then system (1) can be translated into the following random ordinary differential equations (RODEs):

$$
\begin{aligned}
\frac{d x^{(j)}}{d t_{+}} & =F^{(j)}\left(x^{(j)}, O_{t}^{(j)}\right) \\
& :=e^{-O_{t}^{(j)}} f^{(j)}\left(e^{O_{t}^{(j)}} x^{(j)}\right)+O_{t}^{(j)} x^{(j)}, \quad j=1, \ldots, N,
\end{aligned}
$$

where $d x^{(j)} / d t_{+}$is right-hand derivative of $x^{(j)}$ at $t$.

Now we consider the linear coupled RODEs of (6), as follows:

$$
\begin{array}{r}
\frac{d x^{(j)}}{d t_{+}}=F^{(j)}\left(x^{(j)}, O_{t}^{(j)}\right)+v\left(x^{(j-1)}-2 x^{(j)}+x^{(j+1)}\right), \\
j=1, \ldots, N,
\end{array}
$$

with the coupled coefficient $v>0$, where $x^{(0)}=x^{(N)}$ and $x^{(N+1)}=x^{(1)}$. Hence, $(7)$ can be written as the following equivalent MSODEs:

$$
\begin{aligned}
d X_{t}^{(j)}= & f^{(j)}\left(X_{t}^{(j)}\right) d t \\
& +v\left(e^{-\Delta \check{O}_{t}^{(j)}} X_{t}^{(j-1)}-2 X_{t}^{(j)}+e^{-\Delta \widehat{\mathrm{O}}_{t}^{(j)}} X_{t}^{(j+1)}\right) d t \\
& +\sum_{i=1}^{m} c_{i}^{(j)} X_{t}^{(j)} \diamond d L_{t}^{(i)}, \quad j=1, \ldots, N,
\end{aligned}
$$

where $\Delta \check{O}_{t}^{(j)}=O_{t}^{(j)}-O_{t}^{(j-1)}, \Delta \widehat{O}_{t}^{(j)}=O_{t}^{(j)}-O_{t}^{(j+1)}, O_{t}^{(0)}=$ $O_{t}^{(N)}$, and $O_{t}^{(N+1)}=O_{t}^{(1)}$.

For synchronization of solutions (in the sense of Carathéodory [26]) to RODEs system (7), there are two cases: one for any two solutions and the other for components of solutions. When $N=2$, Liu et al. [17] consider both types of synchronization. Under the one-sided dissipative Lipschitz condition (2), they firstly proved that synchronization of any two solutions occurs and the random dynamical system generated by the solution of (7) $N=2$ has a singleton sets random attractor, then they proved that the synchronization between any two components of solutions occurs as the coupled coefficient $v$ tends to infinity. The synchronization result implies that coupled dynamical systems share a dynamical feature in an asymptotic sense. Based on the work of $[15,17]$, we consider the synchronization of solutions of (7) in the case of $N \geq 3$ and obtain the similar results. We show that the random dynamical system (RDS) generated by the solution of the coupled RODEs system (7) has a singleton sets random attractor which implies the synchronization of any two solutions of (7). Moreover, the singleton set random attractor determines a stationary stochastic solution of the equivalently coupled RODEs system (8). We also show that any two solutions of RODEs system (7) converge to a solution $Z(t, \omega)$ of the averaged RODEs as follows:

$$
\frac{d Z}{d t_{+}}=\frac{1}{N} \sum_{j=1}^{N} e^{-O_{t}^{(j)}} f^{(j)}\left(e^{O_{t}^{(j)}} Z\right)+\frac{1}{N} \sum_{j=1}^{N} O_{t}^{(j)} Z,
$$

as the coupling coefficient $v \rightarrow \infty$.

When $\alpha=2$, we have the standard Brownian motion, which the Marcus integral reduces to the Stratonovich stochastic integral, and both types of the synchronization of system (8) have been considered in [15]. It is worth mentioning that the generalization is not trivial because new techniques similar to [15] are needed. We restrict here that $\alpha \in(1,2)$, only in this case, the solutions of the OrnsteinUhlenbech equations based on $\alpha$-stable Lévy noises are stationary, which is crucial to our purpose. When $\alpha \in(0,1)$, dealing with such values of the parameter seems to be a new challenging for us.

The paper is organized as follows. In Section 2, we recall some basic facts on random dynamical systems, and then we give two lemmas which will be frequently used. In Section 3, we show the synchronization of two solutions to the coupled RODEs (7) and obtain the staionary stochastic solution to the equivalent MSODEs (8). In Section 4, we give the 
synchronization of components of solutions to the coupled RODEs (7), which implies that the equivalent MSODEs (8) share the similarly synchronous phenomenon when driven by the same $\alpha$-stable Lévy noises.

\section{Random Dynamical Systems and Auxiliary Lemmas}

We will frequently use the following results.

Lemma 1. There exists a $\left\{\theta_{t}\right\}_{t \in \mathbb{R}}$-invariant subset $\bar{\Omega} \in \mathscr{F}$ of full measure for a.e. $\omega \in \bar{\Omega}$, and the sample paths $\omega(t)$ of $L_{t}$ satisfy

$$
\lim _{t \rightarrow \pm \infty} \frac{\omega(t)}{t}=0, \quad t \in \mathbb{R}
$$

In addition, for $j=1, \ldots, N$, there exist random variables $\bar{O}^{(j)}=O_{t}^{(j)}$ and $T_{\omega}>0$ such that

$$
\begin{gathered}
\bar{O}^{(j)}\left(\theta_{t} \omega\right)=O_{t}^{(j)}(\omega), \quad \lim _{t \rightarrow \pm \infty} \frac{1}{t} \int_{0}^{t} \bar{O}\left(\theta_{s} \omega\right) d s=0, \quad \omega \in \bar{\Omega}, \\
e^{2 \int_{\tau}^{t} O_{s}^{(j)} d s} \leq e^{(L / 2)(t-\tau)} \quad \text { for }-\tau, t>T_{\omega} .
\end{gathered}
$$

Proof. The equalities (10) and (11) can be found in [17, Lemma 2]. By (11), we have $\lim _{t \rightarrow \infty}(1 / t) \int_{0}^{t} O_{s}^{(j)} d s=0$, then there exists $T_{\omega}(1)>0$ such that $\int_{0}^{t} O_{s}^{(j)} d s \leq(L / 4) t$ for $t>T_{\omega}(1)$. Similarly, $\lim _{\tau \rightarrow-\infty}(1 / \tau) \int_{\tau}^{0} O_{s}^{(j)} d s=0$, which implies that there exists $T_{\omega}(2)>0$ such that $\int_{\tau}^{0} O_{s}^{(j)} d s \leq-(L / 4) \tau$ for $\tau<-T_{\omega}(2)$. Denoting $T_{\omega}=\max \left\{T_{\omega}(1), T_{\omega}(2)\right\}$, we have $2 \int_{\tau}^{t} O_{s}^{(j)} d s \leq(L / 2)(t-\tau)$ for $-\tau, t>T_{\omega}$, which completes the proof.

Lemma 2 (Gronwall type inequality). Suppose that $D(t)$ is an $n \times n$ matrix and $\Phi(t)$ and $\Psi(t)$ are $n$-dimensional vectors on $\left[T_{0}, T\right] \quad\left(T \geq T_{0}, T, T_{0} \in \mathbb{R}\right)$ which are sufficiently regular. If the following inequality holds in the componentwise sense:

$$
\frac{d}{d t_{+}} \Phi(t) \leq D(t) \Phi(t)+\Psi(t), \quad t \geq T_{0}
$$

where $\left(d / d t_{+}\right) \Phi(t):=\lim _{h \downarrow 0^{+}}((\Phi(t+h)-\Phi(t)) / h)$ is righthand derivative of $\Phi(t)$, then

$$
\begin{aligned}
\Phi(t) \leq & \exp \left(\int_{T_{0}}^{t} D(s) d s\right) \Phi\left(T_{0}\right) \\
& +\int_{T_{0}}^{t} \exp \left(\int_{\tau}^{t} D(s) d s\right) \Psi(\tau) d \tau, \quad t \geq T_{0} .
\end{aligned}
$$

Proof. See Lemma 2.8 in [27] and the proof of lemma 2.2 in [15].

Proposition 3 (Random attractor for càdlàg RDS (see [16])). Let $(\theta, \varphi)$ be an $R D S$ on $\Omega \times \mathbb{R}^{d}$ and let $\varphi$ be continuous in space but càlàg in time. If there exists a family $\mathscr{B}=\{\mathscr{B}(\omega), \omega \in \Omega\}$ of nonempty measurable compact subsets $\mathscr{B}(\omega)$ of $\mathbb{R}^{d}$ and a $T_{B, \omega} \geq 0$ such that

$$
\varphi\left(t, \theta_{-t} \omega, B\left(\theta_{-t} \omega\right)\right) \subset \mathscr{B}(\omega), \quad \forall t \geq T_{B, \omega},
$$

for all families $B=\{B(\omega), \omega \in \Omega\}$ in a given attracting universe. then, the $\operatorname{RDS}(\theta, \varphi)$ has a random attractor $\mathscr{A}=$ $\{\mathscr{A}(\omega), \omega \in \Omega\}$ with the component subsets defined for each $\omega \in \Omega$ by

$$
\mathscr{A}(\omega)=\bigcap_{s>0} \overline{\bigcup_{t \geq s} \varphi\left(t, \theta_{-t} \omega, B\left(\theta_{-t} \omega\right)\right)} .
$$

Furthermore, if the random attractor consist of singleton sets, that is, $\mathscr{A}(\omega)=\left\{X^{*}(\omega)\right\}$ for some random variable $X^{*}$, then $X_{t}^{*}(\omega)=X_{t}^{*}\left(\theta_{t} \omega\right)$ is a stationary stochastic process.

\section{Synchronization of Two Solutions}

Consider the coupled system (7) with the following initial data:

$$
x^{(j)}(0, \omega)=x_{0}^{(j)}(\omega) \in \mathbb{R}^{d}, \quad \omega \in \Omega, j=1, \ldots, N .
$$

For asymptotic behavior of the difference between two solutions of RODEs system (7) with initial data (17) (omitting to RODEs system (7) for brevity), we get the following:

Lemma 4. For any two solutions $\left(x_{1}^{(1)}(t), x_{1}^{(2)}(t)\right.$ $\left., \ldots, x_{1}^{(N)}(t)\right)^{\mathrm{T}}$ and $\left(x_{2}^{(1)}(t), x_{2}^{(2)}(t), \ldots, x_{2}^{(N)}(t)\right)^{\mathrm{T}}$ of RODEs system (7),

$$
\lim _{t \rightarrow \infty}\left\|x_{1}^{(j)}(t)-x_{2}^{(j)}(t)\right\|=0, \quad j=1, \ldots, N,
$$

that is, all solutions of the coupled RODEs system (7) converge pathwise to each other as time t tends to infinity.

Proof. By the dissipative Lipschitz condition (2), for $j=$ $1, \ldots, N$, we have

$$
\begin{aligned}
\frac{d}{d t_{+}}\left\|x_{1}^{(j)}(t)-x_{2}^{(j)}(t)\right\|^{2} & \\
= & 2\left\langle x_{1}^{(j)}(t)-x_{2}^{(j)}(t), \frac{d}{d t} x_{1}^{(j)}(t)-\frac{d}{d t} x_{2}^{(j)}(t)\right\rangle \\
= & 2 e^{-O_{t}^{(j)}}\left\langle f^{(j)}\left(e^{O_{t}^{(j)}} x_{1}^{(j)}\right)-f^{(j)}\left(e^{O_{t}^{(j)}} x_{2}^{(j)}\right), x_{1}^{(j)}(t)\right. \\
& \left.-x_{2}^{(j)}(t)\right\rangle \\
& \times\left(2 e^{O_{t}^{(j)}}-4 v\right)\left\|x_{1}^{(j)}(t)-x_{2}^{(j)}(t)\right\|^{2} \\
& +2 v\left\langle x_{1}^{(j-1)}(t)-x_{2}^{(j-1)}(t), x_{1}^{(j)}(t)-x_{2}^{(j)}(t)\right\rangle \\
& +2 v\left\langle x_{1}^{(j+1)}(t)-x_{2}^{(j+1)}(t), x_{1}^{(j)}(t)-x_{2}^{(j)}(t)\right\rangle \\
\leq & \left(2 e^{O_{t}^{(j)}}-2 L-2 v\right)\left\|x_{1}^{(j)}(t)-x_{2}^{(j)}(t)\right\|^{2} \\
& +v\left\|x_{1}^{(j-1)}(t)-x_{2}^{(j-1)}(t)\right\|^{2}+v\left\|x_{1}^{(j+1)}(t)-x_{2}^{(j+1)}(t)\right\|^{2} .
\end{aligned}
$$


Define for $t \in \mathbb{R}$,

$$
\begin{aligned}
\mathbf{x}(t)= & \left(\left\|x_{1}^{(1)}(t)-x_{2}^{(1)}(t)\right\|^{2},\left\|x_{1}^{(2)}(t)-x_{2}^{(2)}(t)\right\|^{2}, \ldots,\right. \\
& \left.\left\|x_{1}^{(N)}(t)-x_{2}^{(N)}(t)\right\|^{2}\right)^{\mathrm{T}}, \\
D_{v}(t)= & \left(\begin{array}{ccccc}
\lambda_{v}^{(1)}(t) & v & 0 & \cdots & v \\
v & \lambda_{v}^{(2)}(t) & v & 0 & \cdots \\
0 & v & \lambda_{v}^{(3)}(t) & \ddots & \ddots \\
\vdots & \ddots & \ddots & \ddots & v \\
v & \ldots & 0 & v & \lambda_{v}^{(N)}(t)
\end{array}\right)_{N \times N},
\end{aligned}
$$

where $\lambda_{v}^{(j)}(t)=2 e^{O_{t}^{(j)}}-2 L-2 v, \quad j=1, \ldots, N$. Thus, the differential inequalities can be written as a simple form

$$
\dot{\mathbf{x}}(t) \leq D_{v}(t) \mathbf{x}(t), \quad-\text { componentwise. }
$$

By Lemma 2, it yields from (21) that

$$
\mathbf{x}(t) \leq \exp \left(\int_{0}^{t} D_{v}(t) d s\right) \mathbf{x}(0), \quad \text {-componentwise. }
$$

By [15, Lemma 3.2], we know that for $t \geq T_{\omega}$ defined in Lemma 1 , and $v>0$,

$$
\left\|\exp \left(\int_{0}^{t} D_{v}(t) d s\right) \mathbf{x}(0)\right\| \leq e^{-L t}\|\mathbf{x}(0)\|
$$

which leads to

$$
\lim _{t \rightarrow \infty}\left\|x_{1}^{(j)}(t)-x_{2}^{(j)}(t)\right\|=0, \quad j=1, \ldots, N,
$$

and completes the proof.

Now, we use the theory of random dynamical systems which are generated by stochastic differential equations driven by $\alpha$-stable Lévy noise to find what the solutions of (7) will converge to. Obviously by condition (2) and [16, Lemma 4], we know that the solution

$$
\varphi(t, \omega)=\left(x^{(1)}(t, \omega), x^{(2)}(t, \omega), \ldots, x^{(N)}(t, \omega)\right)^{\mathrm{T}}, \quad \omega \in \Omega,
$$

of system (7) generates a càdlàg $\operatorname{RDS}$ over $\left(\Omega, \mathscr{F}, \mathbb{P},\left(\theta_{t}\right)_{t \in \mathbb{R}}\right)$ with state space $\Omega \times \mathbb{R}^{N d}$.

Then, we have the result for this $\operatorname{RDS} \varphi$.

Theorem 5. Under the dissipative condition of (2), the RDS $\varphi(t, \omega), t \in \mathbb{R}, \omega \in \Omega$, has a singleton sets random attractor given by

$$
\mathscr{A}_{v}(\omega)=\left(\bar{x}_{v}^{(1)}(\omega), \bar{x}_{v}^{(2)}(\omega), \ldots, \bar{x}_{v}^{(N)}(\omega)\right)^{\mathrm{T}},
$$

which implies the synchronization of any two solutions of system (7). Furthermore,

$$
\begin{aligned}
& \left(\bar{x}_{v}^{(1)}\left(\theta_{t} \omega\right) e^{O_{t}^{(1)}(\omega)}, \bar{x}_{v}^{(2)}\left(\theta_{t} \omega\right) e^{O_{t}^{(2)}(\omega)}, \ldots,\right. \\
& \left.\bar{x}_{v}^{(N)}\left(\theta_{t} \omega\right) e^{O_{t}^{(N)}(\omega)}\right)^{\mathrm{T}}
\end{aligned}
$$

is the stationary stochastic solution of the equivalent coupled MSODEs (8).

Proof. For $j=1, \ldots, N$, we have

$$
\begin{aligned}
& \frac{d}{d t}\left\|x^{(j)}(t)\right\|^{2} \\
&=2\left\langle x^{(j)}(t), \frac{d}{d t} x^{(j)}(t)\right\rangle \\
&=2\left\langle e^{-O_{t}^{(j)}} f^{(j)}\left(e^{O_{t}^{(j)}} x^{(j)}(t)\right), x^{(j)}(t)\right\rangle \\
&+2\left\langle e^{O_{t}^{(j)}} x^{(j)}(t), x^{(j)}(t)\right\rangle-4 v\left\|x^{(j)}(t)\right\|^{2} \\
&+2 v\left\langle x^{(j)}(t), x^{(j-1)}(t)\right\rangle+2 v\left\langle x^{(j)}(t), x^{(j+1)}(t)\right\rangle \\
& \leq\left(2 e^{O_{t}^{(j)}}-2 L-2 v\right)\left\|x^{(j)}(t)\right\|^{2}+v\left\|x^{(j-1)}(t)\right\|^{2} \\
&+v\left\|x^{(j+1)}(t)\right\|^{2}+2\left\|x^{(j)}(t)\right\|\left\|f^{(j)}(0)\right\| e^{-O_{t}^{(j)}} \\
& \leq\left(2 e^{O_{t}^{(j)}}-L-2 v\right)\left\|x^{(j)}(t)\right\|^{2}+v\left\|x^{(j-1)}(t)\right\|^{2} \\
&+v\left\|x^{(j+1)}(t)\right\|^{2}+\frac{e^{-2 O_{t}^{(j)}}}{L}\left\|f^{(j)}(0)\right\|^{2} .
\end{aligned}
$$

Analogous to (21), we get

$$
\dot{\mathbf{y}}(t) \leq \widetilde{D}_{v}(t) \mathbf{y}(t)+\mathbf{g}(t),
$$

where $t \in \mathbb{R}$,

$$
\begin{gathered}
\mathbf{y}(t)=\left(\left\|x^{(1)}(t)\right\|^{2},\left\|x^{(2)}(t)\right\|^{2}, \ldots,\left\|x^{(N)}(t)\right\|^{2}\right)^{\mathbf{T}}, \\
\mathbf{g}(t)=\left(\frac{e^{-2 O_{t}^{(1)}}}{L}\left\|f^{(1)}(0)\right\|^{2}, \frac{e^{-2 O_{t}^{(2)}}}{L}\left\|f^{(2)}(0)\right\|^{2}, \ldots,\right. \\
\widetilde{D}_{v}(t)=\left(\begin{array}{ccccc}
\left.\frac{e^{-2 O_{t}^{(N)}}}{L}\left\|f^{(N)}(0)\right\|^{2}\right)^{\mathrm{T}}, & \\
\widetilde{\lambda}_{v}^{(1)}(t) & v & 0 & \ldots & v \\
v & \tilde{\lambda}_{v}^{(2)}(t) & v & 0 & \cdots \\
0 & v & \tilde{\lambda}_{v}^{(3)}(t) & \ddots & \ddots \\
\vdots & \ddots & \ddots & \ddots & v \\
v & \cdots & 0 & v & \tilde{\lambda}_{v}^{(N)}(t)
\end{array}\right)_{N \times N},
\end{gathered}
$$

where $\tilde{\lambda}_{v}^{(j)}(t)=2 e^{O_{t}^{(j)}}-L-2 v, \quad j=1, \ldots, N$. Then by Lemma 2,

$$
\begin{array}{r}
\mathbf{y}(t) \leq \exp \left(\int_{t_{0}}^{t} \widetilde{D}_{v}(t) d s\right) \mathbf{y}\left(t_{0}\right)+\int_{t_{0}}^{t} \\
\exp \left(\int_{\tau}^{t} \widetilde{D}_{v}(t) d s\right) \\
\times \mathbf{g}(\tau) d \tau, \quad t \geq t_{0} .
\end{array}
$$


By (23), we have

$$
\begin{aligned}
\left\|\exp \left(\int_{t_{0}}^{t} \widetilde{D}_{v}(t) d s\right) \mathbf{y}\left(t_{0}\right)\right\| \leq & \exp \left(-\frac{L}{2}\left(t-t_{0}\right)\right) \\
& \times\left\|\mathbf{y}\left(t_{0}\right)\right\|, \quad t \geq t_{0} .
\end{aligned}
$$

Define

$$
\begin{gathered}
\rho_{\nu}(\omega):=\int_{-\infty}^{0} \exp \left(\int_{\tau}^{0} \widetilde{D}_{v}(s) d s\right) \mathbf{g}(\tau) d \tau, \\
R_{v}^{2}(\omega)=1+\left\|\rho_{v}(\omega)\right\|^{2},
\end{gathered}
$$

and let $\mathbb{B}_{v}$ be a random ball in $\mathbb{R}^{N d}$ centered at the origin with radius $R_{v}(\omega)$. Obviously, the infinite integrals on the right hand side of (33) and (34) are well defined by Lemma 1.

For a given attracting universe of tempered random bounded sets $\mathscr{D}$, that is, for any $\omega \in \Omega, B \in \mathscr{D}$, and all $\gamma>0$, we have $\lim _{t \rightarrow \infty} e^{-\gamma t} \sup _{x \in B\left(\theta_{-t} \omega\right)}\|x\|=0$. Note that for all $\gamma>0$, if $\lim _{t \rightarrow \infty} e^{-\gamma t}\left\|\mathbf{y}\left(t_{0}\right)\right\|=0$, then

$$
\sum_{j=1}^{N}\left\|x^{(j)}(0)\right\|^{2}<R_{v}^{2}(\omega) \quad \text { as } t_{0} \longrightarrow-\infty
$$

which implies that the closed random ball $\mathbb{B}_{v}(\omega)$ is a pullback absorbing set at $t=0$ of the càdlàg $\operatorname{RDS} \varphi(t, \omega)$; that is

$$
\varphi\left(t, \theta_{-t} \omega\right) B\left(\theta_{-t} \omega\right) \subset \mathbb{B}_{v}(\omega), \quad \forall t \geq t_{\mathbb{B}_{v}}(\omega)
$$

in the attracting universe $\mathscr{D}$. Hence by Proposition 3 , the coupled system has a random attractor $\mathscr{A}_{v}=\left\{\mathscr{A}_{v}(\omega), \omega \in\right.$ $\Omega$ \} with $\mathscr{A}_{v}(\omega) \subset \mathbb{B}_{v}$ satisfying that $\mathscr{A}_{v}(\omega)$ is compact, $\varphi$ invariant, that is, $\varphi(t, \omega) \mathscr{A}_{v}(\omega)=\mathscr{A}_{v}\left(\theta_{t} \omega\right)$ for all $t \geq 0$, $\omega \in \Omega$, and attracting in $\mathscr{D}$, that is, for all $B \in \mathscr{D}$,

$$
H_{d}^{*}\left(\varphi\left(t, \theta_{-t} \omega, B\left(\theta_{-t} \omega\right)\right), \mathscr{A}_{\nu}(\omega)\right) \longrightarrow 0 \quad \text { as } t \longrightarrow \infty
$$

where $H_{d}^{*}$ is the Hausdorff semidistance on $\mathbb{R}^{N d}$. By Lemma 4, all solutions of (7) converge pathwise to each other; therefor, $\mathscr{A}_{v}(\omega)$ consists of singleton sets, that is,

$$
\mathscr{A}_{v}(\omega)=\left(\bar{x}_{v}^{(1)}(\omega), \bar{x}_{v}^{(2)}(\omega), \ldots, \bar{x}_{v}^{(N)}(\omega)\right)^{\mathrm{T}}
$$

We transform the coupled RODEs (7) back to the coupled MSODEs (8), the corresponding pathwise singleton sets attractor is then equal to

$$
\begin{aligned}
& \left(\bar{x}_{v}^{(1)}\left(\theta_{t} \omega\right) e^{\mathrm{O}_{t}^{(1)}(\omega)}, \bar{x}_{v}^{(2)}\left(\theta_{t} \omega\right) e^{\mathrm{O}_{t}^{(2)}(\omega)}, \ldots,\right. \\
& \left.\bar{x}_{v}^{(N)}\left(\theta_{t} \omega\right) e^{\mathrm{O}_{t}^{(N)}(\omega)}\right)^{\mathrm{T}}
\end{aligned}
$$

which is exactly a stationary stochastic solution of the coupled
MSODEs (8) because the Ornstein-Uhlenbeck process is stationary (see [17]).

\section{Synchronization of the Components of Solutions}

It is known in Section 3 that all solutions of the coupled RODEs system (7) converge pathwise to each other in the future for a fixed positive coupling coefficient $v$. Here, we would like to discuss what will happen to solutions of the coupled RODEs system (7) as $v \rightarrow \infty$. First, we will give some lemmas which play an important role in this section.

Similar to [15, Section 4], we can set up the following estimations. Suppose that $\left(x_{v}^{(1)}(t), x_{v}^{(2)}(t), \ldots, x_{v}^{(N)}(t)\right)^{\mathrm{T}}$ is a solution of the coupled RODEs system (7). For any two different components $x_{v}^{(j)}(t), x_{v}^{(k)}(t)$ of the solution for all $j, k \in$ $\{1,2, \ldots, N\}$

$$
\begin{aligned}
d_{v}^{j, k}(t)= & 2\left\langle x_{v}^{(j)}(t)-x_{v}^{(k)}(t), F^{(j)}\left(x_{v}^{(j)}, O_{t}^{(j)}\right)\right. \\
& \left.-F^{(k)}\left(x_{v}^{(k)}, O_{t}^{(k)}\right)\right\rangle \\
= & 2\left\langle x_{v}^{(j)}(t)-x_{v}^{(k)}(t), e^{-O_{t}^{(j)}} f^{(j)}\left(e^{O_{t}^{(j)}} x_{v}^{(j)}\right)\right. \\
& \left.-e^{-O_{t}^{(k)}} f^{(k)}\left(e^{O_{t}^{(k)}} x_{v}^{(k)}\right)\right\rangle \\
& +2\left\langle x_{v}^{(j)}(t)-x_{v}^{(k)}(t), O_{t}^{(j)} x_{v}^{(j)}(t)-O_{t}^{(k)} x_{v}^{(k)}(t)\right\rangle \\
\leq & 2\left\|x_{v}^{(j)}(t)-x_{v}^{(k)}(t)\right\|\left(e^{-O_{t}^{(j)}}\left\|f^{(j)}\left(e^{O_{t}^{(j)}} x_{v}^{(j)}(t)\right)\right\|\right. \\
& \left.+\left|O_{t}^{(j)}\right|\left\|x_{v}^{(j)}(t)\right\|\right) \\
& +2\left\|x_{v}^{(j)}(t)-x_{v}^{(k)}(t)\right\|\left(e^{-O_{t}^{(k)}}\left\|f^{(k)}\left(e^{O_{t}^{(k)}} x_{v}^{(k)}(t)\right)\right\|\right. \\
& \left.+\left|O_{t}^{(k)}\right|\left\|x_{v}^{(k)}(t)\right\|\right)
\end{aligned}
$$

thus, for fixed $\varrho>0$, we have

$$
\begin{gathered}
-\varrho v\left\|x_{v}^{(j)}(t)-x_{v}^{(k)}(t)\right\|^{2}+d_{v}^{k, j}(t) \\
\leq \frac{1}{v}\left(\frac{4}{\varrho} e^{-2 O_{t}^{(j)}}\left\|f^{(j)}\left(e^{O_{t}^{(j)}} x_{v}^{(j)}(t)\right)\right\|^{2}+\frac{4 v^{2}}{\varrho}\left|O_{t}^{(j)}\right|^{2}\left\|x_{v}^{(j)}(t)\right\|^{2}\right) \\
+\frac{1}{v}\left(\frac{4}{\varrho} e^{-2 O_{t}^{(k)}}\left\|f^{(k)}\left(e^{O_{t}^{(k)}} x_{v}^{(k)}(t)\right)\right\|^{2}\right. \\
\left.+\frac{4 v^{2}}{\varrho}\left|O_{t}^{(k)}\right|^{2}\left\|x_{v}^{(k)}(t)\right\|^{2}\right) .
\end{gathered}
$$


Let

$$
\begin{array}{r}
C_{T_{1}, T_{2}}^{j, k, \varrho}(v, \omega) \\
=\frac{4}{\varrho_{t \in\left[T_{1}, T_{2}\right]}} \sup \left[\left(e^{-2 O_{t}^{(j)}}\left\|f^{(j)}\left(e^{O_{t}^{(j)}} x_{v}^{(j)}(t)\right)\right\|^{2}\right.\right. \\
\left.+\left|O_{t}^{(j)}\right|^{2}\left\|x_{v}^{(j)}(t)\right\|^{2}\right) \\
+\left(e^{-2 O_{t}^{(k)}}\left\|f^{(k)}\left(e^{O_{t}^{(k)}} x_{v}^{(k)}(t)\right)\right\|^{2}\right. \\
\left.\left.+\left|O_{t}^{(k)}\right|^{2}\left\|x_{v}^{(k)}(t)\right\|^{2}\right)\right]
\end{array}
$$

in any bounded interval $\left[T_{1}, T_{2}\right]$. Note that $\rho_{v}(\omega)$ in (33) satisfies

$$
\frac{d}{d v}\left\|\rho_{v}(\omega)\right\|^{2}=2\left\langle\rho_{v}(\omega), \frac{d}{d v} \rho_{v}(\omega)\right\rangle \leq 0
$$

and, consequently, $\rho_{\nu}(\omega) \leq \rho_{1}(\omega)$ for $v \geq 1$. Hence, $C_{T_{1}, T_{2}}^{j, k, \rho}(v, \omega)$ is uniformly bounded in $v$ and

$$
-\varrho v\left\|x_{v}^{(j)}(t)-x_{v}^{(k)}(t)\right\|^{2}+d_{v}^{k, j}(t) \leq \frac{1}{v} C_{T_{1}, T_{2}}^{j, k, \varrho}(v, \omega)
$$

uniformly for $t \in\left[T_{1}, T_{2}\right]$ with

$$
C_{T_{1}, T_{2}}^{j, k, \varrho}(\omega)=\sup _{v \geq 1} C_{T_{1}, T_{2}}^{j, k, \varrho}(v, \omega)
$$

Now let us estimate the difference between any two components of a solution of the coupled RODEs system (7) as $v \rightarrow \infty$.

Lemma 6. Provided condition (2) is satisfied, then any two components of a solution $\left(x_{v}^{(1)}(t), x_{v}^{(2)}(t), \ldots, x_{v}^{(N)}(t)\right)^{\mathrm{T}}$ of the coupled RODEs system (7) uniformly vanish in any bounded time interval when the coupling coefficient $v \rightarrow \infty$; that is, for any bounded interval $\left[T_{1}, T_{2}\right]$ and for all $t \in\left[T_{1}, T_{2}\right]$, it yields

$$
\lim _{v \rightarrow \infty}\left\|x_{v}^{(j)}(t)-x_{v}^{(k)}(t)\right\|=0, \quad \forall j, k \in\{1,2, \ldots, N\} .
$$

Proof. The proof is quite similar to the proof of Lemma 4.2 in [15]. To prove the result, we can equivalently estimate the difference between any two adjacent components only because the first and the last components of the solution are considered to be adjacent. We will notice that only one new term appears in each step which continues the process, except the last step that ends the process.
For the difference of the first part of the solution $\left(x_{v}^{(1)}(t), x_{v}^{(2)}(t), \ldots, x_{v}^{(N)}(t)\right)^{\mathbf{T}}$,

$$
\begin{aligned}
& \frac{d}{d t_{+}}\left\|x_{v}^{(1)}(t)-x_{v}^{(2)}(t)\right\|^{2} \\
&=2\left\langle x_{v}^{(1)}(t)-x_{v}^{(2)}(t), F^{(1)}\left(x_{v}^{(1)}, O_{t}^{(1)}\right)\right. \\
&\left.\quad-F^{(2)}\left(x_{v}^{(2)}, O_{t}^{(2)}\right)\right\rangle \\
& \quad-6 v\left\|x_{v}^{(1)}(t)-x_{v}^{(2)}(t)\right\|^{2} \\
&+ 2 v\left\langle x_{v}^{(1)}(t)-x_{v}^{(2)}(t), x_{v}^{(N)}(t)-x_{v}^{(3)}(t)\right\rangle \\
& \leq-5\left\|x_{v}^{(1)}(t)-x_{v}^{(2)}(t)\right\|^{2}+v\left\|x_{v}^{(N)}(t)-x_{v}^{(3)}(t)\right\|^{2} \\
&+d_{v}^{1,2}(t) \\
& \leq-\delta v\left\|x_{v}^{(1)}(t)-x_{v}^{(2)}(t)\right\|^{2}+v\left\|x_{v}^{(N)}(t)-x_{v}^{(3)}(t)\right\|^{2} \\
&+\frac{1}{v} C_{T_{1}, T_{2}}^{1,2,5}(\omega)
\end{aligned}
$$

uniformly for $t \in\left[T_{1}, T_{2}\right]$ by (44). Here, we can take

$$
\delta= \begin{cases}1-\cos \frac{N \pi}{N+2}, & N \text { is even, } \\ 1-\cos \frac{(N-1) \pi}{N+1}, & N \text { is odd. }\end{cases}
$$

In fact, from [15, Lemma 4.1], we can take any $\delta \in$ $(-2 \cos (N \pi /(N+2)), 2)$ when $N$ is even and any $\delta \in$ $(-2 \cos ((N-1) \pi /(N+1)), 2)$ when $N$ is odd.

We have seen that the estimations in $(47)$ generate $x_{v}^{(3)}(t)-$ $x_{v}^{(N)}(t)$. Now, we have

$$
\begin{aligned}
& \frac{d}{d t_{+}}\left\|x_{v}^{(3)}(t)-x_{v}^{(N)}(t)\right\|^{2} \\
&=2\left\langle x_{v}^{(3)}(t)-x_{v}^{(N)}(t), F^{(3)}\left(x_{v}^{(3)}, O_{t}^{(3)}\right)\right. \\
&\left.\quad-F^{(N)}\left(x_{v}^{(N)}, O_{t}^{(N)}\right)\right\rangle \\
&-4 v\left\|x_{v}^{(3)}(t)-x_{v}^{(N)}(t)\right\|^{2} \\
&+2 v\left\langle x_{v}^{(3)}(t)-x_{v}^{(N)}(t), x_{v}^{(2)}(t)-x_{v}^{(1)}(t)\right\rangle \\
&+ 2 v\left\langle x_{v}^{(3)}(t)-x_{v}^{(N)}(t), x_{v}^{(4)}(t)-x_{v}^{(N-1)}(t)\right\rangle \\
& \leq-\delta v\left\|x_{v}^{(3)}(t)-x_{v}^{(N)}(t)\right\|^{2}+v\left\|x_{v}^{(1)}(t)-x_{v}^{(2)}(t)\right\|^{2} \\
&+ v\left\|x_{v}^{(4)}(t)-x_{v}^{(N-1)}(t)\right\|^{2}+\frac{1}{v} C_{T_{1}, T_{2}}^{3, N, 2-\delta}(\omega)
\end{aligned}
$$

uniformly for $t \in\left[T_{1}, T_{2}\right]$. 
Note that $x_{v}^{(1)}(t)-x_{v}^{(2)}(t)$ has been fixed and $x_{v}^{(4)}(t)-$ $x_{v}^{(N-1)}(t)$ is generated. Similarly, it yields

$$
\begin{aligned}
\frac{d}{d t_{+}}\left\|x_{v}^{(4)}(t)-x_{v}^{(N-1)}(t)\right\|^{2} \\
\leq-\delta v\left\|x_{v}^{(4)}(t)-x_{v}^{(N-1)}(t)\right\|^{2}+v\left\|x_{v}^{(3)}(t)-x_{v}^{(N)}(t)\right\|^{2} \\
\quad+v\left\|x_{v}^{(5)}(t)-x_{v}^{(N-2)}(\mathrm{t})\right\|^{2}+\frac{1}{v} C_{T_{1}, T_{2}}^{4, N-1,2-\delta}(\omega)
\end{aligned}
$$

uniformly for $t \in\left[T_{1}, T_{2}\right]$.

Continuing such estimations, for $j=2,3, \ldots$, we get

$$
\begin{aligned}
\frac{d}{d t_{+}}\left\|x_{v}^{(j+3)}(t)-x_{v}^{(N-j)}(t)\right\|^{2} & \\
\leq & -\delta v\left\|x_{v}^{(j+3)}(t)-x_{v}^{(N-j)}(t)\right\|^{2} \\
& +v\left\|x_{v}^{(j+2)}(t)-x_{v}^{(N-j+1)}(t)\right\|^{2} \\
& +v\left\|x_{v}^{(j+4)}(t)-x_{v}^{(N-j-1)}(t)\right\|^{2}+\frac{1}{v} C_{T_{1}, T_{2}}^{j+3, N-j,-\delta}(\omega)
\end{aligned}
$$

uniformly for $t \in\left[T_{1}, T_{2}\right]$.

We can divide the situation into two cases: $N$ is even and $N$ is odd, which is just as the same as [15] did. When $N$ is even, we can rewrite the inequalities in the matrix form

$$
\dot{\mathbf{u}}(t) \leq \mathbf{H}_{v} \mathbf{u}(t)+\frac{1}{v} \mathbf{C}
$$

uniformly for $t \in\left[T_{1}, T_{2}\right]$, where for $t \in \mathbb{R}$,

$$
\begin{gathered}
\mathbf{u}(t)=\left(\left\|x_{v}^{(1)}(t)-x_{v}^{(2)}(t)\right\|^{2},\left\|x_{v}^{(3)}(t)-x_{v}^{(N)}(t)\right\|^{2}, \ldots,\right. \\
\left.\left\|x_{v}^{(N / 2+1)}(t)-x_{v}^{(N / 2+2)}(t)\right\|^{2}\right)^{\mathrm{T}} \\
\mathbf{C}=\left(C_{T_{1}, T_{2}}^{1,2,5-\delta}(\omega), C_{T_{1}, T_{2}}^{3, N, 2-\delta}(\omega), \ldots,\right. \\
\left.C_{T_{1}, T_{2}}^{N / 2, N+3,2-\delta}(\omega), C_{T_{1}, T_{2}}^{N / 2+1, N / 2+2,5-\delta}(\omega)\right)^{\mathrm{T}}
\end{gathered}
$$

$\operatorname{are}(N / 2)$-dimensional vectors, and

$$
\mathbf{H}_{v}=\left(\begin{array}{ccccc}
-\delta v & v & 0 & \cdots & 0 \\
v & -\delta v & v & \ddots & \vdots \\
0 & v & \ddots & \ddots & 0 \\
\vdots & \ddots & \ddots & -\delta v & v \\
0 & \cdots & 0 & v & -\delta v
\end{array}\right)_{(N / 2) \times(N / 2)} .
$$

By Lemma 2, it follows from (52) that

$$
\mathbf{u}(t) \leq e^{\left(t-t_{0}\right) \mathbf{H}_{v}} \mathbf{u}\left(t_{0}\right)+\frac{1}{v} \int_{t_{0}}^{t} e^{(t-s) \mathbf{H}_{v}} \mathbf{C} d s .
$$

By [15, Lemma 4.1] again, $(1 / v) \mathbf{H}_{v}$ is negative definite, then we have

$$
\left\|e^{\left(t-t_{0}\right) \mathbf{H}_{v}} \mathbf{u}\left(t_{0}\right)\right\| \leq e^{\left(t-t_{0}\right) \mu_{\max }}\left\|\mathbf{u}\left(t_{0}\right)\right\|
$$

where $\mu_{\max }=-\delta-2 \cos (N \pi /(N+2))<0$ is the maximal eigenvalue of $(1 / v) \mathbf{H}_{v}$. Thus, (55) implies that

$$
\begin{gathered}
\mathbf{u}(t) \longrightarrow \mathbf{0} \quad \text { as } v \longrightarrow \infty, \\
\left\|x_{v}^{(1)}(t)-x_{v}^{(2)}(t)\right\|^{2} \longrightarrow 0, \\
\left\|x_{v}^{(N / 2+1)}(t)-x_{v}^{(N / 2+2)}(t)\right\|^{2} \longrightarrow 0
\end{gathered}
$$

uniformly for $t \in\left[T_{1}, T_{2}\right]$ as $v \rightarrow \infty$.

Similarly, when $N$ is odd, we can rewrite the inequalities in the matrix form

$$
\dot{\mathbf{v}}(t) \leq \widetilde{\mathbf{H}}_{v} \mathbf{v}(t)+\frac{1}{v} \widetilde{\mathbf{C}}
$$

uniformly for $t \in\left[T_{1}, T_{2}\right]$, where for $t \in \mathbb{R}$,

$$
\begin{aligned}
\mathbf{v}(t)= & \left(\left\|x_{v}^{(1)}(t)-x_{v}^{(2)}(t)\right\|^{2},\left\|x_{v}^{(3)}(t)-x_{v}^{(N)}(t)\right\|^{2}, \ldots,\right. \\
& \left.\left\|x_{v}^{((N+1) / 2)}(t)-x_{v}^{((N+1) / 2+2)}(t)\right\|^{2}\right), \\
\widetilde{\mathbf{C}}= & \left(C_{T_{1}, T_{2}}^{1,2,5-\delta}(\omega), C_{T_{1}, T_{2}}^{3, N, 2-\delta}(\omega), \ldots,\right. \\
& \left.C_{T_{1}, T_{2}}^{(N-1) / 2,(N+1) / 2+3,2-\delta}(\omega), C_{T_{1}, T_{2}}^{(N+1) / 2,(N+1) / 2+2,5-\delta}(\omega)\right)^{\mathbf{T}}
\end{aligned}
$$

are $((N-1) / 2)$-dimensional vectors, and

$$
\widetilde{\mathbf{H}}_{v}=\left(\begin{array}{ccccc}
-\delta v & v & 0 & \cdots & 0 \\
v & -\delta v & v & \ddots & \vdots \\
0 & v & \ddots & \ddots & 0 \\
\vdots & \ddots & \ddots & -\delta v & v \\
0 & \cdots & 0 & v & -\delta v
\end{array}\right)_{((N-1) / 2) \times((N-1) / 2)}
$$

By Lemma 2, it follows from (58) that

$$
\mathbf{v}(t) \leq e^{\left(t-t_{0}\right) \widetilde{\mathbf{H}}_{v}} \mathbf{v}\left(t_{0}\right)+\frac{1}{v} \int_{t_{0}}^{t} e^{(t-s) \widetilde{\mathbf{H}}_{v}} \widetilde{\mathbf{C}} d s .
$$

Just like the even case, for uniform $t \in\left[T_{1}, T_{2}\right]$, we have

$$
\left\|x_{v}^{(1)}(t)-x_{v}^{(2)}(t)\right\|^{2} \longrightarrow 0, \quad \text { as } v \longrightarrow \infty .
$$

For other adjacent components, the process above can be repeated. Hence, we can draw a conclusion that the difference between any adjacent components of a solution of the coupled RODEs system (7) tends to zero uniformly for $t \in\left[T_{1}, T_{2}\right]$ as the coupling coefficient goes to infinity which completes the proof. 
We know that all components of a solution of system (7) have the same limit uniformly for $t \in\left[T_{1}, T_{2}\right]$ as $v \rightarrow \infty$. Now, we are in the position to find what they converge to.

Lemma 7. If assumptions (2) and (6) hold, the càdlàg random dynamical system $\varphi(t, \omega)$ generated by the solution of the averaged RODEs system

$$
\frac{d Z}{d t_{+}}=\frac{1}{N} \sum_{j=1}^{N} e^{O_{t}^{(j)}} f^{(j)}\left(e^{O_{t}^{(j)}} Z\right)+\frac{1}{N} \sum_{j=1}^{N} O_{t}^{(j)} Z
$$

has a singleton sets random attractor denoted by $\{\bar{Z}(\omega)\}$. Furthermore,

$$
\bar{Z}\left(\theta_{t} \omega\right) \exp \left(\frac{1}{N} \sum_{j=1}^{N} O_{t}^{(j)}(\omega)\right)
$$

is the stationary stochastic solution of the equivalently averaged SODE system

$$
d z=\frac{1}{N} \sum_{j=1}^{N} e^{-\zeta_{t}^{(j)}} f^{(j)}\left(e^{\zeta_{t}^{(j)}} z\right) d t+\frac{1}{N} \sum_{i=1}^{m} \sum_{j=1}^{N} c_{i}^{(j)} z \diamond d L_{t}^{(i)}
$$

where $\zeta_{t}^{(j)}=\sum_{k=1}^{N}\left(O_{t}^{(j)}-O_{t}^{(k)}\right), j=1, \ldots, N$.

Proof. Assume that $Z_{1}(t)$ and $Z_{2}(t)$ are two solutions of (63), we have

$$
\begin{aligned}
& \frac{d}{d t_{+}}\left\|Z_{1}(t)-Z_{2}(t)\right\|^{2} \\
& \quad \leq\left(-2 L+\frac{2}{N} \sum_{j=1}^{N} O_{t}^{(j)}\right)\left\|Z_{1}(t)-Z_{2}(t)\right\|^{2} .
\end{aligned}
$$

It follows from Gronwall's lemma (see [27, Lemma 2.8]) that

$$
\begin{aligned}
& \left\|Z_{1}(t)-Z_{2}(t)\right\|^{2} \\
& \quad \leq e^{-2 t\left(L-(1 / N) \sum_{j=1}^{N}(1 / t) \int_{0}^{t} O_{s}^{(j)} d s\right)}\left\|Z_{1}(0)-Z_{2}(0)\right\|^{2},
\end{aligned}
$$

which implies that

$$
\lim _{t \rightarrow \infty}\left\|Z_{1}(t)-Z_{2}(t)\right\|^{2}=0
$$

Then, all solutions of (63) converge pathwise to each other.

Now, we have to give what they converge to based on the theory of càdlàg random dynamical systems. Let $Z(t)$ be a solution of (63), we get

$$
\begin{array}{r}
\frac{d}{d t_{+}}\|Z(t)\|^{2} \leq( \\
\left.-2 L+\frac{2}{N} \sum_{j=1}^{N} O_{t}^{(j)}\right)\|Z(t)\|^{2} \\
+\frac{1}{L N} \sum_{j=1}^{N} e^{-2 O_{t}^{(j)}}\left\|f^{(j)}(0)\right\|^{2} .
\end{array}
$$

From Gronwall's lemma in [27], again, it yields for $-t_{0}, t>T_{\omega}$,

$$
\begin{aligned}
& \|Z(t)\|^{2} \\
& \leq e^{-L\left(t-t_{0}\right)+(2 / N) \sum_{j=1}^{N} \int_{t_{0}}^{t} O_{s}^{(j)} d s}\left\|Z\left(t_{0}\right)\right\|^{2}+\frac{1}{L N} \\
& \quad \times \sum_{j=1}^{N}\left\|f^{(j)}(0)\right\|^{2} \int_{t_{0}}^{t} e^{-2 O_{\tau}^{(j)}-L(t-\tau)+(2 / N) \sum_{k=1}^{N} \int_{\tau}^{t} O_{s}^{(k)} d s} d \tau .
\end{aligned}
$$

By pathwise pullback convergence with $t_{0} \rightarrow-\infty$, the random closed ball centered as the origin with random radius $\widetilde{R}(\omega)$ is a pullback absorbing set of $\varphi(t, \omega)$, where

$$
\widetilde{R}^{2}(\omega)=1+\frac{1}{L N} \sum_{j=1}^{N}\left\|f^{(j)}(0)\right\|^{2} \int_{-\infty}^{0} e^{L \tau-2 O_{\tau}^{(j)}+(2 / N) \sum_{k=1}^{N} \int_{\tau}^{0} O_{s}^{(k)} d s} d \tau
$$

Obviously, by Lemma 1, the integral defined in the right hand side is well defined.

By Proposition 3, there exists a random attractor $\{\bar{Z}(\omega)\}$ for $\varphi(t, \omega)$. Since all solutions of (63) converge pathwise to each other, the random attractor $\{\bar{Z}(\omega)\}$ is composed of singleton sets.

Note that the averaged RODE (63) is transformed from the averaged SODE (65) by the following transformation:

$$
Z(t, \omega)=z \exp \left(-\frac{1}{N} \sum_{j=1}^{N} O_{t}^{(j)}(\omega)\right)
$$

so the pathwise singleton sets attractor $\bar{Z}\left(\theta_{t} \omega\right) \exp \left((1 / N) \sum_{j=1}^{N} O_{t}^{(j)}(\omega)\right)$ is a stationary solution of the averaged SODE (65) since the Ornstein-Uhlenbeck process is stationary.

Now, we will present another main solution of this work.

Theorem 8. Let

$$
\begin{aligned}
& \left(\bar{x}_{v_{n}}^{(1)}(t, \omega), \bar{x}_{v_{n}}^{(2)}(t, \omega), \ldots, \bar{x}_{v_{n}}^{(N)}(t, \omega)\right)^{\mathrm{T}} \\
& \quad=\left(\bar{x}_{v_{n}}^{(1)}\left(\theta_{t} \omega\right), \bar{x}_{v_{n}}^{(2)}\left(\theta_{t} \omega\right), \ldots, \bar{x}_{v_{n}}^{(N)}\left(\theta_{t} \omega\right)\right)^{\mathrm{T}}
\end{aligned}
$$

be the singleton sets random attractor of the cadlàg random dynamical system $\varphi(t, \omega)$ generated by the solution of RODEs system (7), then

$$
\begin{gathered}
\left(\left(\bar{x}_{v_{n}}^{(1)}(t, \omega), \bar{x}_{v_{n}}^{(2)}(t, \omega), \ldots, \bar{x}_{v_{n}}^{(N)}(t, \omega)\right)^{\mathrm{T}}\right) \\
\longrightarrow(\bar{Z}(t, \omega), \bar{Z}(t, \omega), \ldots, \bar{Z}(t, \omega))^{\mathrm{T}}
\end{gathered}
$$

pathwise uniformly for $t$ belongs to any bounded time interval $\left[T_{1}, T_{2}\right]$ for any sequence $v_{n} \rightarrow \infty$, where $\bar{Z}(t, \omega)=$ $\bar{Z}\left(\theta_{t} \omega\right)$ is the solution of the averaged RODE (63) and $\bar{Z}(\omega)$ is the singleton sets random attractor of the cadlag random dynamical system $\varphi(t, \omega)$ which is generated by the solution of averaged $R O D E(63)$. 
Proof. Define

$$
\bar{Z}_{v}(\omega)=\frac{1}{N} \sum_{j=1}^{N} \bar{x}_{v}^{(j)}(\omega)
$$

where $\left\{\bar{x}_{v}^{(1)}(\omega), \bar{x}_{v}^{(2)}(\omega), \ldots, \bar{x}_{v}^{(N)}(\omega)\right\}$ is the singleton sets random attractor of the càdlàg RDS generated by RODEs system (7). Thus, $\bar{Z}_{v}(t, \omega)=\bar{Z}_{v}\left(\theta_{t} \omega\right)$ satisfies

$$
\begin{aligned}
& \frac{d \bar{Z}_{v}(t, \omega)}{d t_{+}} \\
& \quad=\frac{1}{N} \sum_{j=1}^{N}\left(e^{-O_{t}^{(j)}} f^{(j)}\left(e^{O_{t}^{(j)}} \bar{x}_{v}^{(j)}(t, \omega)\right)+O_{t}^{(j)} \bar{x}_{v}^{(j)}(t, \omega)\right) .
\end{aligned}
$$

Then, we get

$$
\begin{gathered}
\left\|\frac{d \bar{Z}_{v}(t, \omega)}{d t_{+}}\right\|^{2} \leq \frac{2}{N} \sum_{j=1}^{N}\left(e^{-2 O_{t}^{(j)}}\left\|f^{(j)}\left(e^{O_{t}^{(j)}} \bar{x}_{v}^{(j)}(t, \omega)\right)\right\|^{2}\right. \\
\left.+\left|O_{t}^{(j)}\right|^{2}\left\|\bar{x}_{v}^{(j)}(t, \omega)\right\|^{2}\right)
\end{gathered}
$$

by the continuous property of the solutions and the fact that these solutions belong to the compact ball $\mathbb{B}_{1}(\omega)$, it follows that

$$
\sup _{t \in\left[T_{1}, T_{2}\right]}\left\|\frac{d \bar{Z}_{v}(t, \omega)}{d t_{+}}\right\| \leq\left(\frac{2}{N} \sum_{j=1}^{N} \frac{\varrho}{4} \mathbf{C}_{T_{1}, T_{2}}^{j \cdot \bullet}(\omega)\right)^{1 / 2}<\infty .
$$

By the Ascoli-Arzelà theorem in a Skorohod space of bounded time intveral $D\left(\left[T_{1}, T_{2}\right], \mathbb{R}^{d}\right)$ in [23], there exists a subsequence $v_{n_{k}} \rightarrow \infty$ such that $\bar{Z}_{v_{n_{k}}}(t, \omega)$ converges to $\bar{Z}(t, \omega)$ as $n_{k} \rightarrow \infty$.

Since difference between any two components of a solution of the coupled RODEs system (7) tends to zero uniformly for $t \in\left[T_{1}, T_{2}\right]$ as $v \rightarrow \infty$, from (75), we have

$$
\begin{aligned}
\bar{x}_{v_{n_{k}}}^{(j)}(t, \omega)= & \bar{Z}_{v_{n_{k}}}(t, \omega) \\
& +\frac{1}{N} \sum_{j^{\prime} \neq j j^{\prime \prime} \neq j^{\prime}}\left(\bar{x}_{v_{n_{k}}}^{\left(j^{\prime \prime}\right)}(t, \omega)-\bar{x}_{v_{n_{k}}}^{\left(j^{\prime}\right)}(t, \omega)\right) \\
& \longrightarrow \bar{Z}(t, \omega)
\end{aligned}
$$

uniformly for $t \in\left[T_{1}, T_{2}\right]$ as $v_{n_{k}} \rightarrow \infty$ for $j=1, \ldots, N$. Furthermore, it follows from (76) that, for $t \geq T_{1}$,

$$
\begin{gathered}
\bar{Z}_{v}(t, \omega)=\bar{Z}_{v}\left(T_{1}, \omega\right)+\frac{1}{N} \sum_{j=1}^{N} \int_{T_{1}}^{t}\left(e^{-O_{s}^{(j)}} f^{(j)}\left(e^{O_{s}^{(j)}} \bar{x}_{v}^{(j)}(s, \omega)\right)\right. \\
\left.+O_{s}^{(j)} \bar{x}_{v}^{(j)}(s, \omega)\right) d s
\end{gathered}
$$

Thus,

$$
\begin{gathered}
\bar{Z}(t, \omega)=\bar{Z}\left(T_{1}, \omega\right)+\frac{1}{N} \sum_{j=1}^{N} \int_{T_{1}}^{t}\left(e^{-O_{s}^{(j)}} f^{(j)}\left(e^{O_{s}^{(j)}} \bar{Z}(s, \omega)\right)\right. \\
\left.+O_{s}^{(j)} \bar{Z}(s, \omega)\right) d s
\end{gathered}
$$

uniformly for $t \in\left[T_{1}, T_{2}\right]$ as $v_{n_{k}} \rightarrow \infty$, which implies that $\bar{Z}_{v}(s, \omega)$ solves $\operatorname{RODE}(63)$. Then, we note that all possible sequences of $\bar{Z}_{v_{n_{k}}}(t, \omega)$ converge to the same limit $\bar{Z}(t, \omega)$ uniformly for $t \in\left[T_{1}, T_{2}\right]$ as $\nu_{n} \rightarrow \infty$. Since the $\operatorname{RDS} \varphi$ generated by the solutions of RODE (63) has a singleton sets random attractor $\{\bar{Z}(\omega)\}$, the stationary stochastic process $\bar{Z}\left(\theta_{t} \omega\right)$ must be equal to $\bar{Z}(t, \omega)$, that is, $\bar{Z}(t, \omega)=\bar{Z}\left(\theta_{t} \omega\right)$, which completes the proof.

As an obvious result of Theorem 8, we get the following.

\section{Corollary 9.}

$$
\begin{gathered}
\left(\left(\bar{x}_{v}^{(1)}(t, \omega), \bar{x}_{v}^{(2)}(t, \omega), \ldots, \bar{x}_{v}^{(N)}(t, \omega)\right)^{\mathrm{T}}\right) \\
\longrightarrow(\bar{Z}(t, \omega), \bar{Z}(t, \omega), \ldots, \bar{Z}(t, \omega))^{\mathrm{T}}
\end{gathered}
$$

in Skorohod metric pathwise uniformly for $t \in\left[T_{1}, T_{2}\right]$ as $v \rightarrow$ $\infty$.

Remark 10. The results in this paper hold just in almost everywhere because $\omega \in \bar{\Omega}$ in Lemma 1 , and we still use $\Omega$ instead of $\bar{\Omega}$.

Remark 11. Although the same results hold when the systems perturbed by non-Gaussian noises (see, e.g., [17] and this paper for $\alpha$-stable Lévy noises and $[16,18]$ for additive Lévy noises), there exists some difference between dealing with such stochastic systems when driven by Bronian motions and Lévy motions. Firstly, to some extent, the cases of Lévy noises have more general sense than the Bronian motions. For example, when $\alpha=2$, the Lévy noise is the standard Brownian motion and the Marcus integral is reduced to the Stratonovich stochastic integral, that is, the case of multiplicative white noise (see $[11,15])$. Here we only are restricted to $1<\alpha<2$. Secondly, We need to consider the càdlàg functions in the Skorohod metric, which are different from the continuous cases in the metric under the compactopen topology. Last but not least, we have to consider the solutions in the sense of Carthéodory and the right hand derivatives.

\section{Acknowledgments}

The author would like to thank the anonymous referees for their helpful comments and suggestions which largely improved the quality of the paper. This work is partially supported by NSF of China under Grant no. 11071165, Guangxi Provincial Department of Research Project (201010LX166), 
and Program to Sponsor Teams for Innovation in the Construction of Talent Highlands in Guangxi Institutions of Higher Learning under Grant no. [2011] 47.

\section{References}

[1] V. S. Afraimovich, S.-N. Chow, and J. K. Hale, "Synchronization in lattices of coupled oscillators," Physica D, vol. 103, no. 1-4, pp. 442-451, 1997.

[2] V. S. Afraimovich and W.-W. Lin, "Synchronization in lattices of coupled oscillators with Neumann/periodic boundary conditions," Dynamics and Stability of Systems, vol. 13, no. 3, pp. 237-264, 1998.

[3] V. S. Afraĭmovich, N. N. Verichev, and M. I. Rabinovich, "Stochastic synchronization of oscillations in dissipative systems," Izvestiya Vysshikh Uchebnykh Zavedeniu, vol. 29, no. 9, pp. 1050-1060, 1986.

[4] S. Strogatz, Sync: The Emerging Science of Spontaneous Order, Hyperion Press, 2003.

[5] A. Pikovsky, M. Rosenblum, and J. Kurths, Synchronization, A Universal Concept in Nonlinear Sciences, Cambridge University Press, 2001.

[6] L. Glass, "Synchronization and rhythmic processes in physiology," Nature, vol. 410, pp. 277-284, 2001.

[7] V. S. Afraimovich and H. M. Rodrigues, "Uniform dissipativeness and synchronization of nonau-tonomous equation," in Proceedings of the International Conference on Differential Equations, pp. 3-17, World Scientific, Lisbon, Portugal, 1995.

[8] P. E. Kloeden, "Synchronization of nonautonomous dynamical systems," Electronic Journal of Differential Equations, vol. 39, pp. 1-10, 2003.

[9] A. N. Carvalho, H. M. Rodrigues, and T. DThlotko, "Upper semicontinuity of attractors and synchronization," Journal of Mathematical Analysis and Applications, vol. 220, no. 1, pp. 1341, 1998.

[10] H. M. Rodrigues, "Abstract methods for synchronization and applications," Applicable Analysis, vol. 62, no. 3-4, pp. 263-296, 1996.

[11] T. Caraballo and P. E. Kloeden, "The persistence of synchronization under environmental noise," Proceedings of The Royal Society of London A, vol. 461, no. 2059, pp. 2257-2267, 2005.

[12] T. Caraballo, P. E. Kloeden, and A. Neuenkirch, "Synchronization of systems with multiplicative noise," Stochastics and Dynamics, vol. 8, no. 1, pp. 139-154, 2008.

[13] T. Caraballo, I. D. Chueshov, and P. E. Kloeden, "Synchronization of a stochastic reaction-diffusion system on a thin twolayer domain," SIAM Journal on Mathematical Analysis, vol. 38, no. 5, pp. 1489-1507, 2007.

[14] I. Chueshov and B. Schmalfuß, "Master-slave synchronization and invariant manifolds for coupled stochastic systems," Journal of Mathematical Physics, vol. 51, no. 10, Article ID 102702, 23 pages, 2010.

[15] Z. W. Shen, S. F. Zhou, and X. Y. Han, "Synchronization of coupled stochastic systems with multiplicative noise," Stochastics and Dynamics, vol. 10, no. 3, pp. 407-428, 2010.

[16] X. M. Liu, J. Q. Duan, J. C. Liu, and P. E. Kloeden, "Synchronization of dissipative dynamical systems driven by non-Gaussian Lévy noises," International Journal of Stochastic Analysis, vol. 2010, Article ID 502803, 13 pages, 2010.
[17] X. M. Liu, J. Q. Duan, J. C. Liu, and P. E. Kloeden, "Synchronization of systems of Marcus canonical equations driven by $\alpha$ stable noises," Nonlinear Analysis, vol. 11, no. 5, pp. 3437-3445, 2010.

[18] A. H. Gu, "Synchronization of coupled stochastic systems driven by non-Gaussian Lévy noises", Stochastic and Dynamics, submitted.

[19] D. Applebaum, Lévy Processes and Stochastic Calculus, Cambridge University Press, Cambridge, UK, 2004.

[20] S. Peszat and J. Zabczyk, Stochastic Partial Differential Equations with Léevy Processes, Cambridge University Press, Cambridge, UK, 2007.

[21] K.-I. Sato, Lévy Processes and Infinitely Divisible Distributions, vol. 68 of Cambridge Studies in Advanced Mathematics, Cambridge University Press, Cambridge, UK, 1999.

[22] G. Samorodnitsky and M. S. Taqqu, Stable Non-Gaussian Random Processes, Chapman \& Hall, New York, NY, USA, 1994.

[23] P. Billingsley, Convergence of Probability Measures, John Wiley \& Sons, New York, NY, USA, 1968.

[24] L. Arnold, Random Dynamical Systems, Springer Monographs in Mathematics, Springer, 1998.

[25] S. I. Marcus, "Modeling and approximation of stochastic differential equations driven by semimartingales," Stochastics, vol. 4, no. 3, pp. 223-245, 1981.

[26] M. Errami, F. Russo, and P. Vallois, "Itô's formula for $C^{1, \lambda}$ functions of a càdlàg process and related calculus," Probability Theory and Related Fields, vol. 122, no. 2, pp. 191-221, 2002.

[27] J. C. Robinson, Infinite-Dimensional Dynamical Systems, Cambridge University Press, Cambridge, UK, 2001. 


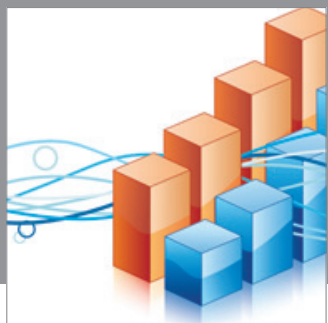

Advances in

Operations Research

mansans

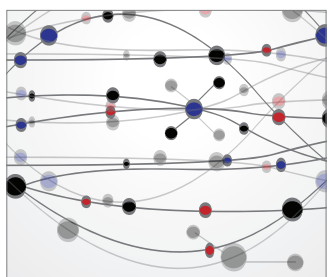

The Scientific World Journal
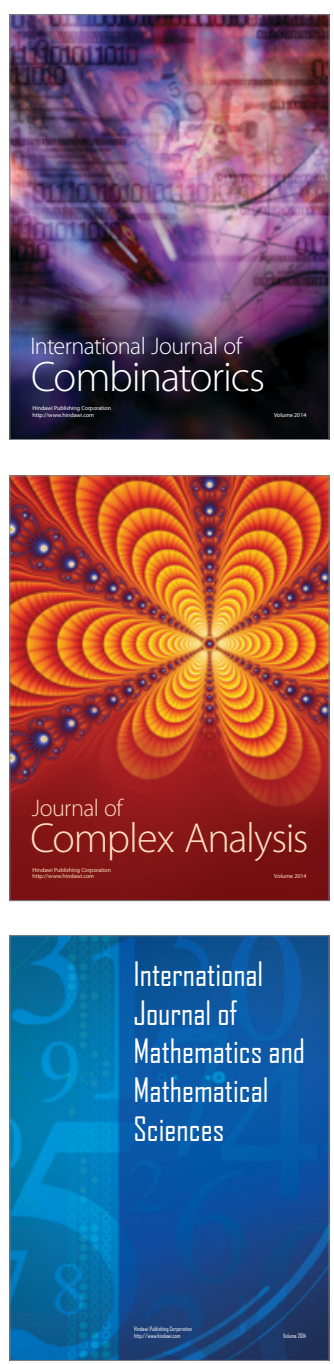
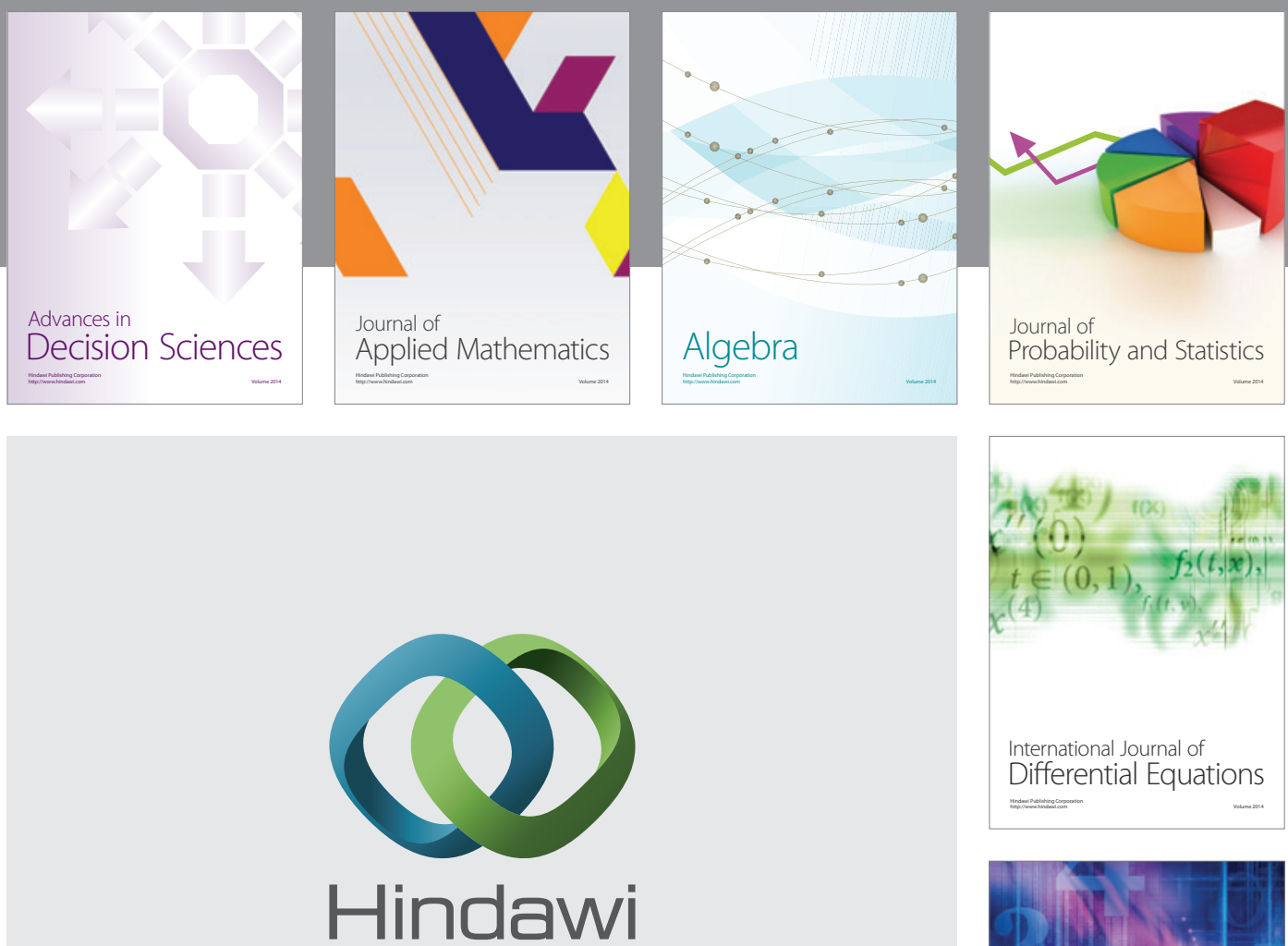

Submit your manuscripts at http://www.hindawi.com
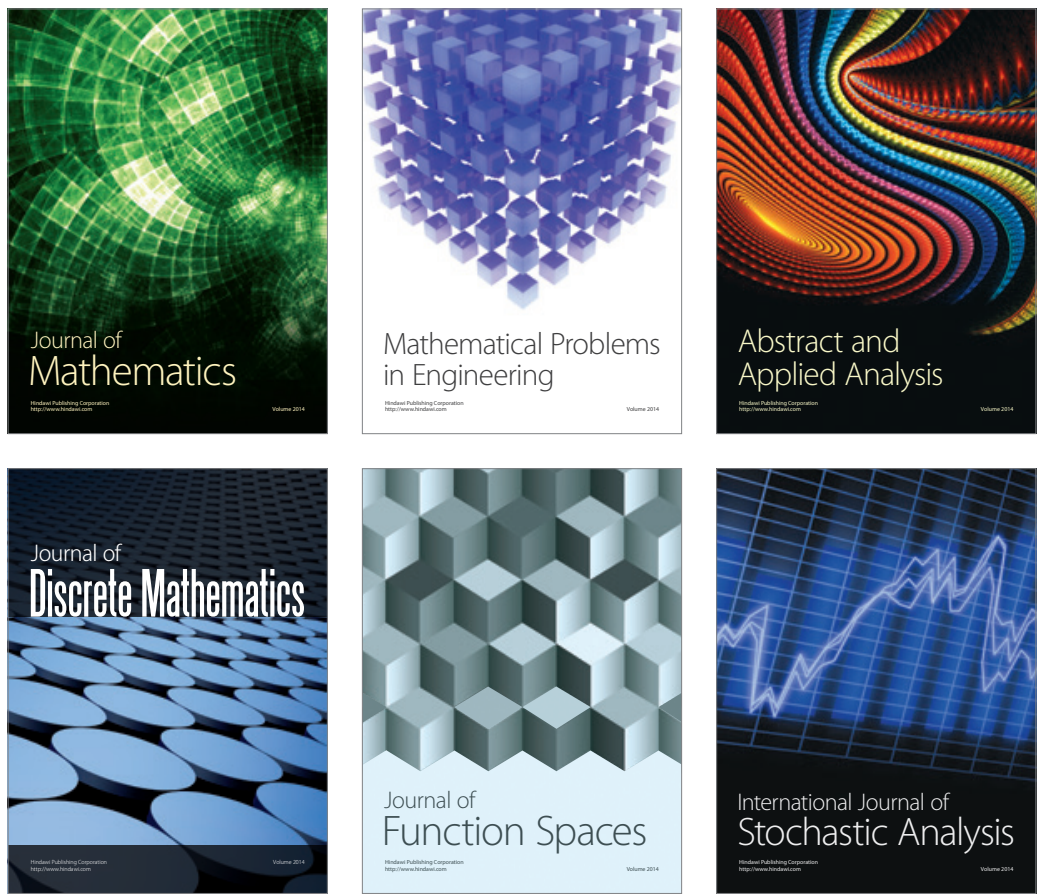

Journal of

Function Spaces

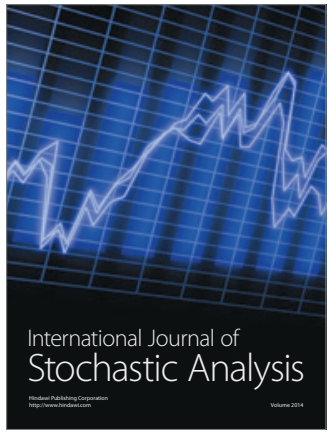

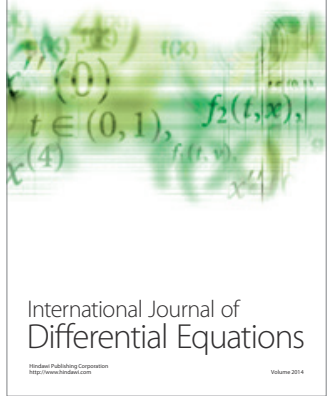
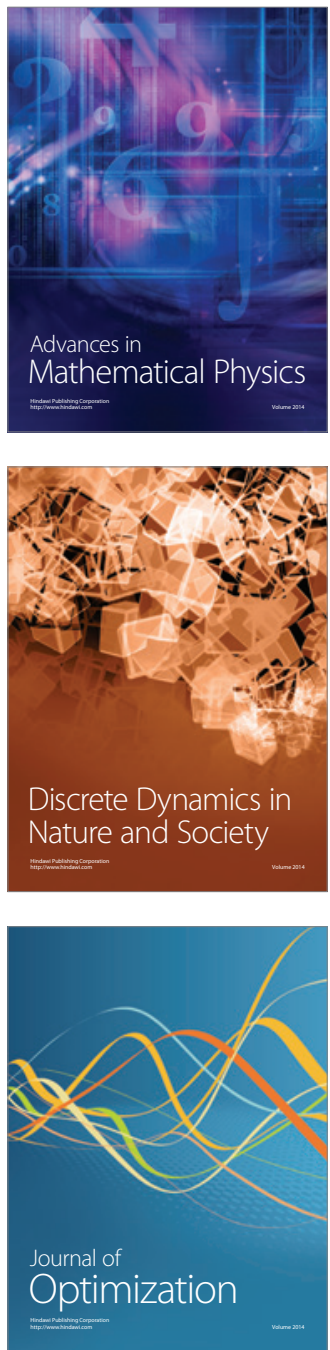